International Journal of Engineering \& Technology, $7(2.8)(2018) 340-344$
International Journal of Engineering \& Technology
SPC
Website: www.sciencepubco.com/index.php/IJET
Research Paper

\title{
Microstrip line Antenna Fabrication Material
}

\author{
Vimlesh Singh $^{1 *}$, Priyanka Bansal ${ }^{2}$, P.K. Singhal ${ }^{3}$ \\ ${ }^{1,2}$ Department of ECE, FET, MRIU, Faridabad \\ ${ }^{3}$ Department of ECE, MITS, Gwalior \\ *Corresponding author E-mail: vimlesh.fet@mriu.edu.in
}

\begin{abstract}
This paper presents an extensive survey of electromagnetic materials used for antenna fabrication, which find application in Civilian life as well as defense life. When a densely packed microwave integrated circuit is designed, it requires protection from higher power transient because of specific polarization and frequency response. To meet specification of such kind of microwave circuits it is desired to exploit properties of fabricating materials, which are not found in nature but can be prepared with specific proportion of chemical element combination. This study provides in-depth responses of materials toward electromagnetic wave's characteristics such as dielectric, flexible electronics, electrical and thermal properties, which have vast potential in communication engineering.
\end{abstract}

Keyword: Material, Electromagnetic, Microwave, Dielectric

\section{Introduction}

With rapid technological growth, it is highly indebted to the innovation of better engineering material. For plentiful growth in engineering areas such as electronic/electrical products, aerospace projects, nuclear engineering and numerous other related activities based on material technology. Materials are always a basis of any industry from last many years simple materials are used in manufacturing of engineering goods. These common materials such as metal, plastic, ceramic, alloy and many more has distinct physical and chemical attribution. Technology decides how to utilize properties of any different applications.

In past years, advent usage of simple material facilitates state of art technological improvement in creation as well as synthesis of new material from the combination of two or more materials. These combinations carry characteristics of all constituents that can be utilized as per the requirement of material. In this modern scenario, as the technology upgrade, it helps in a more accurate microscopic analysis of the morsel of materials, which enable to assess the behavioral characteristics in a better manner.

Material engineering is itself a rapid developing domain in engineering science so a realization of new material makes this subject more challenging for future prospect. This domain of science $\&$ technology has been potential to facilitate modern category of material, which can be used for unique applications. This paper provides study of application oriented materials that conjectures multiple components together for optimal usage and flexibility [11$12]$.

\section{Important Parameter of Antenna Materials}

Electromagnetic properties of microwave material depend on mode of propagation at microscopic level. In microwave material electromagnetic force field occur phenomenally in two situations:

i. Field force due to static charge because of fundament particles such as proton \& electron, which is equal in magnitude and follow all basic rules.

ii. Field force due to dynamic charge because of motion of charge particle from one region to different region of depth of penetration (skin depth).

Macroscopic level characteristics of microwave material extremely depend on following parameter:

1. Basic parameter: dielectric constant $(\varepsilon)$, permeability $(\mu) \&$ Conductivity of material $(\sigma)$

2. Medium (linear or non-liner/ homogenous or heterogeneous/ isotropic or anisotropic)

3. Material response toward frequency of operation.

Table 1: Sub- classification of dielectric, conducting \& magnetic material [15]

\begin{tabular}{|l|l|l|}
\hline Dielectric Material & Conducting Material & $\begin{array}{l}\text { Magnetic } \\
\text { Material }\end{array}$ \\
\hline Perfect dielectric $(\sigma=0)$ & $\begin{array}{l}\text { Perfect conductor }(\sigma \\
=\infty)\end{array}$ & $\begin{array}{l}\text { Diamagnetic }(\mu \\
=1)\end{array}$ \\
\hline $\begin{array}{l}\text { Imperfect (Lossy) dielectric } \\
(\sigma \neq 0)\end{array}$ & Conductor & Paramagnetic \\
\hline
\end{tabular}

Dielectric \& Magnetic property of material explain electric polarization due to applied electric field, this linear polarization is due to applied potential for this proportionate constant known as 
permittivity of material.

Permittivity,

$\varepsilon_{r}=\frac{\varepsilon}{\varepsilon_{0}}$

$\varepsilon=\varepsilon^{\prime}+\varepsilon^{\prime \prime}$

This $\varepsilon_{r}$ creates confusion because ' $r$ ' stands for relative term. This dielectric factor is associated with the "loss factor" because of energy absorption by the material. Another factor related to this is "Loss Tangent." It calculates the energy absorption level of material in an imaginary \& complex plane.

Loss tangent, $\quad \tan \delta=\frac{\varepsilon^{\prime \prime}}{\varepsilon^{\prime}}$

Dielectric properties of material depend on: Dipole Reorientation and Conduction. Dipole reorientation occurs because of asymmetry across the crystal structure. This cause dipole molecule fragmentation that cancels the effect of electric field and electron shift to the nuclei, thus spatial charge created. Dielectric properties of material are also dependent on external condition such as temperature and pressure.

Magnetic property of material depends on an externally exerted magnetic field which interacts with the magnetic energy incident to the material surface. This is also related to permeability of material. Generally natural material has a slight or zero magnetic response, and their absolute magnetic permeability is equivalent to free space permeability.
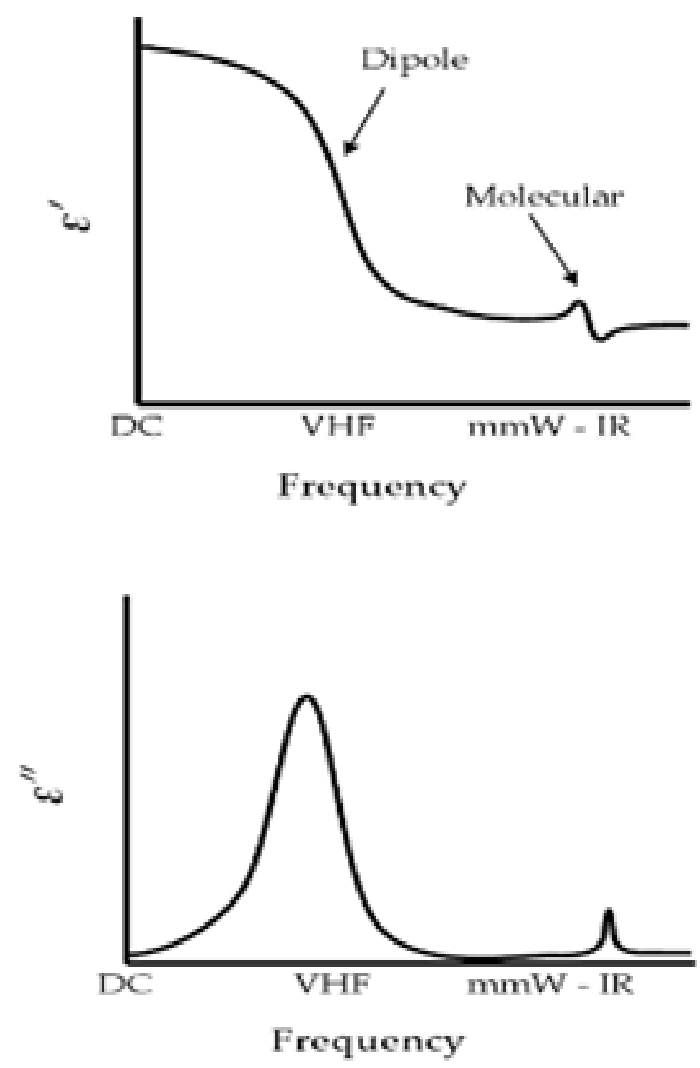

Fig.1: National Real \& Imaginary permittivity of Dispersive Material [15]

\subsection{Materials for Microwave Application}

Material plays major role in technological advancement of electronic engineering domain. It makes communication fast and accurate Printed antenna engineering is one of the essential elements of any communication network because of easy ease of fabrication in respect to size as well as cost. Small size antennas have number of benefits perhaps it has some limitation. Those limitations can be improved by using different techniques of antenna analysis.

Nowadays, printed antennas are preferred because of its compact size and easy fabrication by PCB (printed -circuit -board) design methods. To design any printed circuit board antenna appropriate substrate is selecting in such a way that material provides mechanical strength to the antenna. Dielectric of substrate also plays an important role for moderating electrical properties of antenna because surface waves produced on substrate dielectric extract total available power for radiation in space wave. Cost of production of antenna is also affected by choice of substrate material. Therefore, choosing and evaluation of correct substrate are one necessary domain of antenna design. So for design of any antenna as per characteristics initially material is selected on which test antenna will be fabricated. The main parameter used for design of any antenna is its dielectric constant, thickness of laminate, dissipation factor, thermal coefficient and many other tradeoff. With any shrink in dimension of antenna, using PCB fabrication changes various parameter substrate materials such as structural and tensile strength, resistance to chemical, impact resistance, flexibility, bond ability, machinability and many others are important. The various types of substrate material is used for different application. A very wide range of substrate material of PTFE (poly tetra fluoro ethylene), polyphenylene, polystyrene, sapphire, alumina, ferromagnetic, semiconductor substrate and rutile used for different applications. The choice of substrate material depends on its application because there is no ideal substrate material. For design of low frequency applications high dielectric constants materials are used to keep dimensions of antenna small [1] \& [2].

\subsection{Ceramic Substrates}

Ceramic fabrication technique provides clarification to be high interconnect density, Compactness \& higher frequency of operation. Ceramic substrate is available in LTCC (low Temperature Co-fired Ceramic) \& HTCC (High-Temperature Temperature Co-fired Ceramic). There are various antennas are fabricated on the ceramic substrate. The most common ceramic materials used for design of antenna circuits are alumina (Al2O3) because it has minute loss and very minuscule dispersion with frequency. It has extremely high thermal conductivity \& strength. A very wide range of ceramic materials are available with dielectric constant of 20 to 150 [1].

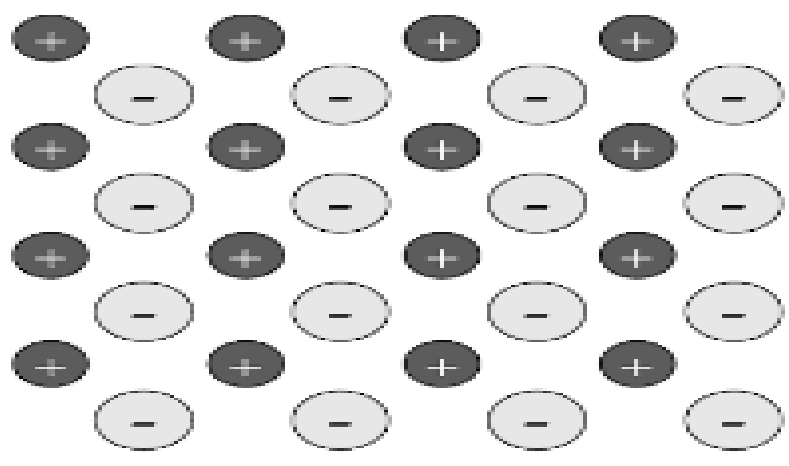

Fig. 2: Charge distribution in ceramic material

In 2009, M. Henry, C. E. Free, B. S. Izqueirdo, J. Batchelor, and P. Young presented paper based on Photo-Image Based Ceramic Substrate with title "Millimeter-Wave Wave Substrate Integrated Waveguide Antennas: Design and Fabrication Analysis"[5]. This antenna structure is used at high millimeter-wave frequencies. Photo-imageable process consists of four major steps: First, a thick film screen is printed on a substrate at room temperature. Second 
print is exposed to ultra-violet through photo pattern chroma mask which is polymerized and harden in the exposed area. Third remove unexposed material and dried by air spray.

In 2010, A. Rashidian, M. Tayfeh Aligodarz, D. M. Klymyshyn, M. Boerner, and J. Mohr present paper based on Poly Ceramic substrate with Composite Material with title "Photo definable Micro composites for Antenna Applications" [7]. This paper is based on design of antenna by using another polyceramic composite material. This polymer- ceramic material is used as the dielectric of highenergy density with an embedded capacitor. This material is also used in medical applications to develop a high-performance transducer to convert mechanical energy of composite material in its corresponding electrical energy. The various types of polymer binders are also added in composite substrate to enhance characteristics of substrate material. Polydimethylsiloxane (PDMS) is elastic by nature where as polyethylene is having very good dielectric properties. SU-8 is common epoxy negative photo resist material used by Soviet government for ground attack aircraft. It can be spread over thickness $1 \mu \mathrm{m}$ to $300 \mu \mathrm{m}$. Its biocompatible substrate material used it can most widely be used in bio-MEMS.

\subsection{Semiconductor Substrate}

This category of material is known as terahertz material, which fabricated by semiconductor material. For design of antenna and passive circuits, its semi-insulating materials are used. This semiinsulating material has high resistivity semiconductor materials such as silicon ( $\mathrm{Si}$ ) or gallium arsenide (GaAs). To design an antenna for microwave application the dimensional size of substrate is a very small monolithic integrated circuit.

In 2010 Aidin Mehdipour, Iosif D. Rosca, Abdel-Razik Sebak, Christopher W. Trueman and Suong. V. Hoa presented a paper on Carbon Nano Material with tilte " Full-Composite Fractal Antenna Using Carbon Nano tubes for Multiband Wireless Applications"[8]. This paper is based on carbon element for design of antenna. These carbon atoms are in forms of the cluster which are carbon nanotube (CNT). To design the proposed antenna substrate of these CNT materials is fabricated on both sides of sheet. This carbon -fiber composites (CFC) are widely used by aerospace industry because of its good strength, light weight and low cost. CFC is most widely used in RFID antenna where as RCCF for ultra wideband (UWB) applications.

In 2012 Aidin Mehdipour, Abdel R. Sebak , Christopher W. Trueman, Iosif. D. Rosca and Suong V. Hoa presented paper on "Advanced conductive carbon-fiber composite materials for antenna and microwave applications"[11]. This paper is based on carbonfiber composite material used for microwave applications. There are two chief category of CFC material one is RCCF, and another is CNT in which RCCF is preferred for several applications because of anisotropic behavior of it. The two main kinds of CFC material were discovered by Concordia Center for Composite (CONCOM) lab. In this paper antenna designed by RCCF material, which has resin reinforced by fibers with specific orientation. The conductivity of RCCF each layer is higher along fiber but less in perpendicular direction. To enhance conductivity of RCCF composite a very small fraction of Multiwall carbon nanotube add in RCCF material. $\mathrm{RCCF}$ is also used to obtain reconfigurable characteristics of antenna.

\subsection{Ferrimagnetic Metalized Substrate}

These ferrite substrates are anisotropic by nature. Ferrite substrate has relative permittivity in the range of 9 to 16 with low loss tangent. For Ferrimagnetic material at frequency of $10 \mathrm{GHz}$ its loss tangent is .001. The frequency of operation of microwave antenna designed by ferrite substrate depends on the biased magnetic field. Ferrite is magnetic superstrates material, which is frequently used as the dielectric substrate to enhance the performance of an array or its element. The ferrite substrate material can be easily used in design of antenna if it does not affect radiation characteristics of antenna. In 2011 Steven E. Morris, Yakup Bayram, Lanlin Zhang, Zheyu Wang, Max Shtein, and John L. Volakis presented a paper on Metal Fiber Substrate with jtitle "High-Strength, Metalized Fibers for Conformal Load Bearing Antenna Applications" [10]. This paper is based on load-bearing material for microwave applications, which is good for electrical conducting configuration. These materials are metal- coated, high-strength fiber of multi-filament coated with Kevlar yarns. These Kevlar yarns have weaving flexibility, volumetric, stretchable so provide a high-frequency response in comparison to the rigid ceramic(Rogers TMM4) substrate. These UAV also requires higher mechanical stress, strain induced cracking, elongation and hysteresis response. Therefore, these antenna structures needs an alternative conducting medium for achieve required RF performance. The various E-textile technologies are considered for improving flexibility and resistance of antenna structure. To achieve high electrically conductive metal like copper and silver is used but these have poor tensile strength. So in this metal fiber strength - to - weight ratio of conductive fiber optimization is main constrain. For normalizing the parametric performance of these composite fibers is coated or increase the thickness of metal fiber. Kevlar 49 yarns fabrications are in four steps such as: Initially polymer film etch by sodium hydroxide. Secondly, this is followed by "RTM solution C" sodium hypophosphite than it is followed by "RTM solution B" by palladium chloride in copper solution in "copper solution $\mathrm{A}$ " and "copper solution B" finally in copper sulfate and $\mathrm{HCHO}$ (formaldehyde). The yarns formed by his technique are then twisted to achieve maximum strength because this twisting increases friction between adjacent fibers so load is distributed across the length. This study conclude that performance of metal coil fiber is better than E-fiber(polymer-ceramic composite) in frequency range of $0.1 \mathrm{GHz}$ to $1 \mathrm{GHz}$.

\subsection{Synthetic Substrates}

The various organic materials are used as substrate material for design of antenna. These materials include benzene ring such as Teflon, polyolefin, polystyrene and polyphenylene has low los tangent and permittivity. Mechanical strength and stability of these material are not very good, so they used when no alternative material is present.

In 2013 M. Tayfeh Aligodarz, A. Rashidian2, D. M. Klymyshyn, M. Schulz, M. Boerner,T Hanemann, P. Meyer, and J. Mohr presented a paper on Polyester-Styrene Ceramic Nano Composite Substrate with title "Polyester-styrene/Ceramic Nano composites for Antenna Applications"[13]. This paper is based on polymer polyester-styrene composite of higher permittivity Barium Titanate. The usage of high permittivity ceramic material increases relative permittivity of composite material at microwave frequencies. The material used for design of antenna is prepared by polyester resin diluted with 20 percent/weight styrene to reduce viscosity of base polymer material. The ceramic content required for this contains Barium Titanate in powder form of minute size. Ceramic material added to the unsaturated mixture also consists of small quantity of a dispenser to increase homogeneity of the composite material. Permittivity of this new composite material is compared with pure polyester- styrene with the help of a material analyzer, and it is found that permittivity of composite material improved. It is also concluded that with addition of Barium - titanate enhances permittivity where as the dielectric constant and loss tangent remains same. 


\subsection{Composite Material Substrates}

These are the material which manufactured by combine characteristics of various basic materials to enhance electrical and mechanical characteristics so these materials are known as composite materials. These materials are the combination of basic material so its mechanical characteristics are modified, and permittivity adjusted as per applications. Composite substrate material is available in permittivity of the range 2.1 to 10 and loss tangent of .0005 to .002 at frequency of $10 \mathrm{GHz}$. These materials having a good mechanical strength so allowing for machining work and fabrication of antenna possible by standard PCB design technique. The list of few composite substrate materials is given below [6 \& 16-17].

In 2009 Lara J.Martin, Sooliam Ooi, Daniela Staiculescu, Michael D. Hill, C. P. Wong and Manos M. Tentzeris presented a paper on Tetra Composite Substrate with title "Effect of Permittivity and Permeability of a flexible Magnetic Composite Material on the Performance and Miniaturization Capability of Planar Antennas for RFID and Wearable Wireless Applications" [6]. The main problem with magnetic composite material is its magnetic loss, which must be low for targeted frequency of application. So to reduce this issue a flexible composite material of Co-hexaferrite $(\mathrm{Co} 2 \mathrm{Z})$ in the matrix of Silicon material was developed. The key finding of paper is its permeability because of a small amount of permeability provide substantial capability of minimization. The magnetic composite material developed by Z- phase cobalt hexaferrite in the hexagonal form this size on paper was 45-150 micrometer. The composite material consists of Co2Z partials in Dow Corning Sylgard Silicone 184 matrices. Co2Z particles consist of blending oxides and carbonates of composition of stoichiometric elements (Ba3Co2Fe24O41)Magnetic composite material was formulated by adding $40 \%$ volume of ferrite particle in silicon into wet power. Material properties such as dielectric constant, permeability, loss tangent are analyzed.

\subsection{Low Loss Tangent substrates}

Substrate materials used for design of microwave antenna are having good radiation efficiency. These materials have very good electrical performance for various commercial applications but its cost is too high. So, it related to mass production of these printed antenna (includes the cost of substrate and connector). FR4 and Epoxy are most widely used low cost substrate material, which is easy availability and fabrication. It used for frequency below $1 \mathrm{GHz}$ because of high loss tangent and variable dielectric constant.

\subsection{Metamaterial}

Metamaterial is an artificial material which not found in nature. These elements have the capability of controlling and adjusting light, sound and other physical parameter. These elements are clouds of multiple or individual elements arrange in conventional fashion such as plastic or metals. Compositional properties of Metamaterial are different from its base materials, but they arrange in specific shape. The specific shape, size, orientation, geometry and arrangement of element effect electromagnetic radiation in an artificial manner. These materials having sub-wavelength and reverse refractive index capability. So these materials are also known as negative index material and good optical resolution beyond the limit of conventional lens. The potential usage of Metamaterial is in sensor detection, smart solar power management, remote aerospace, infrastructure monitoring, public safety, highfrequency army application, lens in high -gain antenna, earthquake shielding structure and ultrasonic sensors. Metamaterial are also used in interdisciplinary field of engineering and science.
In 2005 Douglas J. Kern Douglas H. Werner, Agostino Monorchio, Luigi Lanuzza, and Michael J. Wilhelm presented a paper on Eclectronic Band Gap magnetic Meta material with title "The Design synthesis of Multiband Artificial Magnetic Conductors Using High Impedance Frequency Selective Surfaces" [3]. This paper is based on introduction of different design method for multiresonance artificial magnetic conducting surfaces (AMC). Paper investigates multiband characteristics with the help of electronic band gap (EBG) AMC. The structure of a prefect band gap Metamaterial surface consists of both sided periodic metallic patterns.

In 2010 Radu Malureanu, Andrei Lavrinenko, David G.Cooke, Peter Uhd Jepsen and S.Xiao, L.Zhou presented a paper on Terahetz Time domain Metamaterial with title "Transmission and Reflection Properties of Terahertz Fractal Metamaterials" [9]. This paper is based on Terahertz time- domain analysis of reflection properties of fractal Metamaterial geometry. The antenna structures were fabricated on highly resistive $\mathrm{Si}$ substrate. Substrate geometry was coated with 50 nanometers thin gold $(\mathrm{Au})$ layer by the process of optical lithography then a layer of 4 micrometers thin $\mathrm{Ni}$ was grown electrochemically and selective removal of gold form this layer takes place.

In 2013 Trong N. Duong, Jayanti Venkatarama and Zhaolin Lu presented paper on Nanoplasmonics metamaterial "Enhancement of Ambient Energy Haresting Using a Metamaterial Lens" [14]. This paper is based on harvesting of Radio frequency energy by Metamaterial lens. The lens structure was designed by Nanoplasmonics and matematerial researcher that stack 65 printed circuit board together, and a copper wire etched on top facing of each board. The antenna was designed for Koch curve upto second iteration. The beam focusing capability of this antenna is up to $5.49 \mathrm{GHz}$ by the horn antenna. The test of antenna for the frequency of $2.5 \mathrm{GHz}$ and $5.49 \mathrm{GHz}$ antenna structure was simulated by HFSS and optimization done by genetic algorithm. The major application of this lens is in biomedical implanted electronics.

\section{Conclusion}

This study provides an integrated solution of microwave materials for various applications in different area of science \& technology. Molecular characteristics of material changes with change in parameter of materials, this also affects physical \& chemical properties of materials.

Material which provides low dielectric constant between the range of 1 to 2 are porous by nature, and their permittivity is one. So, they reduce loss from surface wave. It also increases the resonance frequency of operation with high gain and acceptable bandwidth.

Higher and stable the dielectric constant of material increases the absorbing property material which can be used as wearable material of different biomedical applications. Thickness of material dielectric is another crucial parameter in microwave material because it maximizes the bandwidth of planner structure, but it impacts efficiency of antenna.

\section{References}

[1] Ramesh Garg, Prakash Bartia, Inder Bahl and Apisak Ittipiboon, "Microstrip Antenna Design Handbook", Artech House Inc. Norwood, MA, pp. 168, 253-316, 759-767, 2001.

[2] Panoutsopoulos, B., "Printed circuit fractal antennas", presented in Consumer Electronics, 2003, ICCE-2003 IEEE International Conference on , pp. $288-289,2003$.

[3] Douglas J. Kern, Douglas H. Werner,Agostino Monorchio, Luigi Lanuzza, and Michael J.Wilhelm, "The Design Synthesis of Multiband Artificial Magnetic Conductors Using High Impedance 
Frequency Selective Surfaces" IEEE Transactions Antennas and Propagation, Vol.53, No.1, pp. 8-17, 2005.

[4] Leila Yousefi and Omar M. Ramahi, "Experimental Retrieval of the Effective Parameters of Artificial Magnetic Materials based on a the Microstrip Line Method" Antennas and Propagation Society International Symposium-2008, pp.1-4, 2008.

[5] M. Henry, C. E. Free, B. S. Izqueirdo, J. Batchelor, and P. Young, "Millimeter Wave Substrate Integrated Waveguide Antennas: Design and Fabrication Analysis" IEEETransactions on Advanced Packaging, Vol.32, No.1, pp.93-100, 2009.

[6] Lara J. Martin, Sooliam Ooi, Daniela Staiculescu, Michael D. Hill, C. P. Wong, and

[7] Manos M. Tentzeris, "Effect of Permittivity and Permeability of a Flexible MagneticComposite Material on the Performance and Miniaturization Capability of Planar Antennas for RFID and Wearable Wireless Applications" IEEE Transactions on Components andPackaging Technologies, Vol.32, No.4, pp.849858, 2009.

[8] Rashidian, M. Tayfeh Aligodarz, D. M. Klymyshyn, M. Boerner, and J. Mohr, "Photodefinable Microcomposites for Antenna Applications" Antennas and Propagation Society International Symposium (APSURSI), pp. 1- 4, 2010.

[9] Aidin Mehdipour, Iosif D. Rosca, Abdel-Razik Sebak, Christopher W. Trueman, andSuong. V. Hoa, "Full-Composite Fractal Antenna Using Carbon Nanotubes for

[10] Multiband Wireless Applications" IEEE Antenna and Wireless Propagation Letters, Vol. 9, pp. 891-894, 2010.

[11] Radu Malureanu, Andrei Lavrinenko, David G. Cooke, and Peter Uhd Jepsen S. Xiao, L.Zhou (2010), "Transmission and Reflection Properties of Terahertz Fractal Metamaterials"Lasers and ElectroOptics (CLEO) and Quantum Electronics and Laser Science Conference (QELS), pp. 1-2, 2010.

[12] Steven E. Morris, Yakup Bayram, Lanlin Zhang, Zheyu Wang, Max Shtein, and John L.Volakis, "High-Strength, Metalized Fibers for Conformal Load Bearing Antenna Applications" IEEE Transactions Antennas and Propagation, Vol. 59, No.9, pp. 34583466, 2011

[13] Aidin Mehdipour, Abdel R. Sebak, Christopher W. Trueman, Iosif. D. Rosca and Suong V.Hoa , "Advanced conductive carbon fiber composite materials for antenna and microwave applications" Antennas and Propagation Society International Symposium

(APSURSI), pp. 1-2, 2012.

[14] John Schultz, Focused Beam Methods: Measuring Microwave Materials in Free Space Technology \& Engineering pp.1-18, 2012.

[15] M. Tayfeh Aligodarz, A. Rashidian, D. M. Klymyshyn, M. Schulz, M. Boerner, T. Hanemann, P. Meyer, and J. Mohr , "Polyesterstyrene/Ceramic Nanocomposites forAntenna Applications" Antennas and Propagation Society International Symposium

(APSURSI), Orlando, FL, pp. 1906 - 1907, 2013.

[16] Trong N. Duong, Jayanti Venkataraman and Zhaolin Lu, "Enhancement of Ambient Energy Harvesting Using a Metamaterial Lens" Antennas and Propagation Society International Symposium (APSURSI), pp. 962-963, 2013.

[17] Deborah D. L. Chung "Composite Materials: Functional Materials for Modern Technologies", Springer, pp.1-10, 2013.

[18] Vimlesh Verma \& P.K Singhal "Q-Arc Micro strip Fractal Antenna - in International Journal of Research in Antennas and Microwave Engineering in, Vol.02, Issue 01;pp.570-573, Jan- Feb 2013.

[19] Vimlesh Verma \& P.K Singhal "Electronic Band Gap Substrate Micro strip Dual band Fractal Antenna" in International Journal of Electronics \& Communication Technology Vol. 4 Issue 1,pp.405410, Jan-March 2013.

[20] Meka Bharadwaj, Hari Kishore "Enhanced Launch-Off-Capture Testing Using BIST Designs" Journal of Engineering and Applied Sciences, ISSN No: 1816-949X, Vol No.12, Issue No.3, page: 636643, April 2017.

[21] T. Padmapriya and V. Saminadan, "Priority based fair resource allocation and Admission Control Technique for Multi-user Multiclass downlink Traffic in LTE-Advanced Networks", International Journal of Advanced Research, vol.5, no.1, pp.1633-1641, January 2017.

[22] S.V.Manikanthan and K.srividhya "An Android based secure access control using ARM and cloud computing", Published in: Electronics and Communication Systems (ICECS), 2015 2nd
International Conference on 26-27 Feb. 2015, Publisher: IEEE,DOI:10.1109/ECS.2015.7124833. 